\title{
Value of hyperventilation in pattern-reversal visual evoked potentials
}

\author{
J BEDNARIK, O NOVOTNY \\ From the Department of Neurology, University of J E Purkyně, Brno, Czechoslovakia
}

SUMMARY The effects of a standard 3 minutes' hyperventilation on the full-field pattern-reversal visual evoked potential (VEP) were studied in 33 normal subjects, 30 definite multiple sclerosis patients and in twenty-five patients with abnormal VEPs due to either tumourous compression of the anterior visual pathways or optic atrophy of other origin. Significantly greater reductions in P100 latency occurred in multiple sclerosis patients in comparison with controls $(p<0.05)$. This change appeared to be specific for demyelinative type of lesion, for it was not found in cases with other types of pathology. Hyperventilation also increased the sensitivity of visual pathway impairment detection in multiple sclerosis.

Transient improvement in neurological deficits after hyperventilation has been reported in patients with central nervous system demyelination. ${ }^{1}$ The effect of hyperventilation is believed to be mediated through the reduction in $\mathrm{pCO}_{2}$ and the rise in extracelluar $\mathrm{pH}$, as well as through the reduction in serum ionised calcium level causing the change in nerve fibre excitability and the safety factor for transmission in central fibres. ${ }^{1-4}$ Later the effects of hyperventilation on pattern-reversal VEP have been studied and transient and significant reduction in P100 latency after hyperventilation was detected in definite multiple sclerosis patients with clinical and/or VEP evidence of visual pathway impairment. ${ }^{5}$ Duration of hyperventilation in that study was controlled by actual carbon dioxide pressure in the blood. In our study we have investigated the effects of a standard 3 minutes' hyperventilation on full-field pattern-reversal VEP in patients with definite multiple sclerosis and with visual pathway impairment of other origins, to assess the validity of this simple activation method in routine VEP testing.

Address for reprint requests: Dr J Bednarik, Department of Neurology, University of J E Purkyně, Brno 656 91, Czechoslovakia.

Received 23 September 1988 and in revised form 3 March 1989. Accepted 10 March 1989

\section{Subjects and methods}

\section{Subjects}

Thirty-three volunteers, either students or staff members, aged from 20 to 50 years, 14 men and 19 women, all of whom had normal visual acuity, served as control subjects. Thirty consecutive patients with multiple sclerosis who fulfilled Poser' ${ }^{6}$ criteria for either clinically or laboratory supported definite multiple sclerosis, aged from 18 to 55 years, 9 men and 21 women, formed the second group irrespective of clinical or VEP evidence of visual pathway impairment. The third group we examined consisted of 25 patients with VEP abnormalities due to either tumourous compression of the anterior visual pathways (17 patients) or optic atrophy of non-tumourous origin (7 patients).

\section{Method}

Monocular VEPs to full-field stimulation were recorded with round surface mid-occipital electrodes placed at $\mathrm{O}_{2}$ and inion, both referred to $F_{z}$ (10-20 system). The black and white checkerboard stimulus was displayed on a standard TV monitor (Phillips) by a visual pattern generator (Dantec). The luminance and contrast (0.9) were kept constant and the pattern was reversed in $20 \mathrm{~ms}$ every $500 \mathrm{~ms}$. The visual angle of the screen was $14^{\circ}$ by $11^{\circ}$, with each check subtending $40^{\circ}$ of visual angle. Usually 200 runs were averaged using an electromyograph (Neuromatic $2000 \mathrm{C}$, Dantec) with a low frequency filter of $250 \mathrm{~Hz}$. The procedure was repeated until at least two reproducible responses were obtained. The response with higher amplitude of P100 component (mostly from $\mathrm{O}_{2}$ ) was chosen for further investigation and calculation of normal data. 


\section{Activation procedure}

After baseline monocular recording in each subject the eye which gave more abnormal, but still clearly recognisable and reproducible response with macular P100 component, was selected for testing of hyperventilation effect (usually the one with longer P100 latency). In cases with bifid (W) shape of the main positive deflection as well as in patients with central scotomas additional vertical half-field stimulation $\left(10^{\circ}\right.$ by $14^{\circ}$ ) was performed and VEPs from the ipsilateral electrodes positioned $5 \mathrm{~cm}$ lateral to $\mathrm{O}_{2}$ were recorded, in order not to confuse the P100 macular component with the paramacular one. ${ }^{7-9}$ The subjects were then instructed to breathe deeply for 3 minutes, then the selected full-field was recorded immediately, while the subject continued in slight hyperventilation.

\section{Results}

For the normal limit for absolute P100 latency and interlocular P100 latency difference we used the mean $+3 \mathrm{SD}$ (in our control group the normal limits were $112 \mathrm{~ms}$ and $5.5 \mathrm{~ms}$, respectively) and for the normal limit for the absolute P100 amplitude we used the lowest recorded response in normal subjects ${ }^{10}(3 \cdot 5 \mu \mathrm{V}$ in our normal group). Using these normal limits in our multiple sclerosis group we found abnormal VEPs in 23 cases $(76 \cdot 7 \%)$.

In 33 control subjects hyperventilation resulted in the mean fall in P100 latency of $-0.51 \mathrm{~ms}$ (SD $-1.41 \mathrm{~ms}$ ) (range +2 to $-3 \mathrm{~ms}$ ) (fig 1 ). The normal limit for the post-hyperventilation latency reduction at $3 \mathrm{SD}$ level was calculated as $-4.75 \mathrm{~ms}$. In the multiple sclerosis group the mean fall in P100 latency was $-3.08 \mathrm{~ms}$ (fig 2 ) which was significantly greater than in normal subjects $(p<0.01)$. The normal limit for P100 latency reduction was exceeded in 10 cases $(33.3 \%)$, including two patients with normal prehyperventilation VEPs (see fig 2). The absolute P100 latency of these patients' tested eyes were just within normal limit (111 and $110 \mathrm{~ms}$, respectively). Ophthalmological findings in these two patients were normal

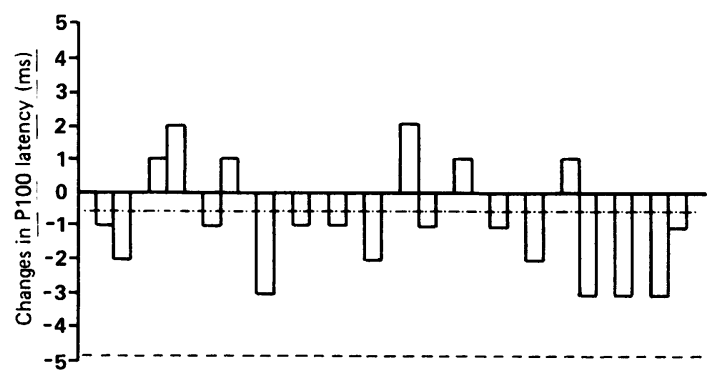

Fig 1 Post-hyperventilation changes in P100 latency in 33 control subjects (each column represents one tested subject) with the mean fall of $-0.51 \mathrm{~ms}$ (dashed and dotted line). The normal limit (mean 3 SD) for post-hyperventilation latency changes was calculated as $-4.75 \mathrm{~ms}$ (dashed line).

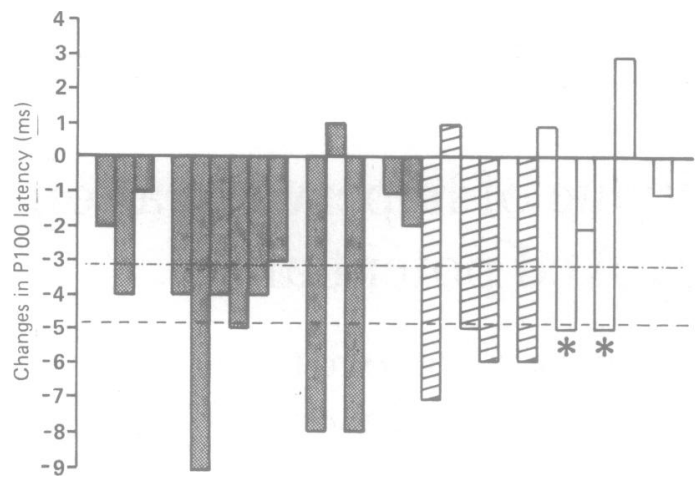

Fig 2 Post-hyperventilation changes in P100 latency in 30 definite multiple sclerosis patients with the mean fall of $-3.08 \mathrm{~ms}$ (dashed and dotted line). Double-hatched columns represent cases with abnormal pre-hyperventilation absolute P100 latency in tested eye, single-hatched columns represent cases with normal absolute but abnormal interocular latency difference, and empty columns represent cases with normal pre-hyperventilation VEP findings. Two patients from the latter group showing post-hyperventilation latency change that exceeded the normal limit (dashed line), are marked with asterisks.

and there was a history of vague transient visual complaints in one of them.

There was no clear correlation between the degree of latency change and the pre-hyperventilation latency. Duration of visual symptoms had no significant influence on the degree of post-hyperventilation latency reduction. In the non-multiple sclerosis group the mean fall in latency was $-0.59 \mathrm{~ms}$, range +2 to $-4 \mathrm{~ms}$; the difference between this group and the normal subjects was not significant.

Changes in P100 amplitude after hyperventilation were bidirectional in both control subjects (range +33 to $-33 \%$ ), and multiple sclerosis and non-multiple sclerosis patients. The differences between these three groups were not significant.

\section{Discussion}

Hyperventilation is known to bring about a transient improvement in neurological deficits in multiple sclerosis, ' which is very similar to that caused by hypocalcaemia and alcalosis, ${ }^{1}$ mild hypothermia, administration of phosphates ${ }^{12}$ and 4-aminopyridine. ${ }^{13}$ The effect of hyperventilation is probably mediated through the reduction in $\mathrm{pCO}_{2}$ and the rise in extracellular $\mathrm{pH}$ and perhaps also through the secondary reduction in serum ionised calcium level ${ }^{1}$ enhancing axonal excitability and increasing the safety factor for transmission ${ }^{2}$ in demyelinated borderline conducting and blocked nerve fibres. The improvement of visual signs was documented in 
definite multiple sclerosis patients with visual impairment, in which hyperventilation caused significant reduction in P100 latency. ${ }^{5}$ Either half-field or fullfield VEPs were tested and the duration of hyperventilation was controlled by actual carbon dioxide pressure until $\mathrm{pCO}_{2}$ decreased below $25 \mathrm{~mm} \mathrm{Hg}$.

We wished to know if the using of constant duration hyperventilation that could be used in routine clinical testing would have the same effect. As in most patients in the above study $\mathrm{pCO}_{2}$ decreased below $25 \mathrm{~mm} \mathrm{Hg}$ after 1.5 minutes of hyperventilation, ${ }^{5}$ we used the duration of 3 minutes. Our results confirmed previously published findings and showed the same influence of hyperventilation on P100 latency in definitive multiple sclerosis patients as a group. The sensitivity of the post-hyperventilation P100 latency reduction in the detection of demyelinative type of lesion was $33 \%$, which was lower than that of either absolute P100 latency or interlocular latency difference. ${ }^{10}$ However, in two definite multiple sclerosis patients with normal ophthalmological and prehyperventilation VEP findings we found latency reduction exceeding slightly the normal limit for posthyperventilation latency changes and thus the activation procedure increased the sensitivity of VEP testing from $76.7 \%$ before hyperventilation to $83.3 \%$ which represents the increase of $6.5 \%$. It is possible to suppose demyelinative involvement causing slight prolongation in $\mathrm{P} 100$ latency that falls within the range from +2.5 to $+3 \mathrm{SD}$, as in our two patients. As most authors prefer using normal limits at 3 SD level to avoid false positive results, ${ }^{10}$ some cases with slight impairment can show VEP values still within normal limits. Hyperventilation thus seems to be useful especially in cases with borderline VEP findings.

In spite of the fact that in compressive lesions of visual pathways local demyelinative component is supposed to participate in visual involvement, ${ }^{14}$ there were no significant latency reductions in our patients. It is difficult to explain the differences in posthyperventilation latency changes in different patients with demyelinative involvement of visual pathways and even in patients with sclerosis multiplex. The extent of a demyelinating involvement cannot be the only factor, as the cases with the longest latencies did not always show the largest reductions and in compressive lesions we did not find significant decrement in spite of a moderate prolongation of P100 latency in some of them. We were not able to confirm the possibility that recent demyelinative lesions are more susceptible to various influences which change conduction conditions. Larger and multiple sites of demyelination, as present in multiple sclerosis and optic neuritis, are probably necessary for the effect of hyperventilation on visual fibres to become expressed.

However, hyperventilation can be helpful in cases of abnormal VEP findings when the possibility of a visual pathway of a demyelinative type is in doubt. The changes in P100 latency contrast with the lack of a consistent pattern of amplitude changes in multiple sclerosis. This is surprising in view of the fact that visual acuity in optic neuritis correlates with the amplitude of P100 component, ${ }^{15}$ and the reversal of conduction $\mathrm{V}$ block in demyelinative fibres is the most probable mechanism of the effect of hyperventilation on VEP. ${ }^{5}$

\section{References}

1 Davis FA, Becker FO, Michael FA, Sorensen E. Effect of intravenous sodium bicarbonate, disodium editate ( $\mathrm{Na}_{2}$ EDTA) and hyperventilation on visual and oculomotor signs in multiple sclerosis. $J$ Neurol Neurosurg Psychiatry 1970;33:723-32.

2 Davis FA, Schauf CL. The pathophysiology of multiple sclerosis: a theoretical model. In: Klawans HL, ed. Models of Neurological Diseases. Amsterdam: Excerpta medica 1974:84-107.

3 Schain RJ. Cerebrospinal fluid cation levels. Arch Neurol 1964;11:330-3.

4 Rogart RB, Ritchie JM. Pathophysiology of conduction in demyelinated nerve fibres. In: Morell P, ed. Myelin. New York: Plenum Press 1077:353-82.

5 Davies HD, Carroll WM, Mastaglia FL. Effects of hyperventilation on pattern-reversal visual evoked potentials in patients with demyelination. $J$ Neurol Neurosurg Psychiatry 1986;49:1392-6.

6 Poser ChM, Paty DW, Scheinberg L, et al. New diagnostic criteria for multiple sclerosis. Ann Neurol 1983;13:277-31.

7 Halliday AM, Barrett G, Blumhardt LD, Kriss A. The macular and paramacular subcomponents of the pattern evoked response. In: Lehmann D, Callaway E, eds. Human Evoked Potentials. London: Plenum Publishing 1979:135-51.

8 Blumhardt LD, Barrett G, Halliday AM, Kriss A. The effect of experimental "scotomata" on the ipsilateral and contralateral responses to pattern-reversal in one half-field. Electroencephalogr Clin Neurophysiol 1978;45:376-92.

9 Carroll WM, Mastaglia FL, Thickbroom GW. The topography and origin of central and peripheral subcomponents of the half field pattern reversal visual evoked potentials. Neurosci Lett 1985;Suppl 19:S50.

10 Chiappa KH. Evoked Potentials in Clinical Medicine. New York: Raven Press 1983:1-24.

11 Watson CW. Effect of lowering body temperature on the symptoms and signs of multiple sclerosis. $N$ Engl J Med 1959;261:1253-9.

12 Becker FO, Michael JA, Davis FA. Acute effects of oral phosphate on visual function in multiple sclerosis. Neurology 1974;24:601-7.

13 Stefoski D, Davis FA, Faut M, Schauf CL. 4-Aminopyridine improves clinical signs in multiple sclerosis. Ann Neurol 1987;21:71-7.

14 Halliday AM, Halliday E, Kriss A, McDonald WI, Mushin J. The pattern-evoked potentials in compression of the anterior visual pathways. Brain 1976;99:357-74.

15 Halliday AM. Visual evoked potentials in demyelinating disease. In: Waxman SG, Ritchie JM, eds. Demyelinating Disease: Basic and Clinical Electrophysiology. New York: Raven Press 1981:201-15. 